\title{
Fractional Ablative Radio-Frequency Resurfacing in Asian and Caucasian Skin: A Novel Method for Deep Radiofrequency Fractional Skin Rejuvenation
}

\author{
R. Stephen Mulholland ${ }^{*}$, D. H. Ahn², Michael Kreindel ${ }^{3}$, Malcolm Paul $^{4}$ \\ ${ }^{1}$ Private Plastic Surgery Practice, Toronto, Canada; ${ }^{2}$ Daeyoung Plastic Surgery Practice, Seoul, South Korea; ${ }^{3}$ Invasix Ltd., Toronto, \\ Canada; ${ }^{4}$ Department of Surgery, Aesthetic and Plastic Institute, University of California, Irvine, California. \\ Email: *mulhollandmd@spamedica.com
}

Received May $9^{\text {th }}, 2012$; revised June $13^{\text {th }}, 2012$; accepted June $28^{\text {th }}, 2012$

\begin{abstract}
This paper reports the clinical experience of a multi-center, multiple physician trial with a novel fractional radiofrequency ablative skin resurfacing and rejuvenation device (Fractora, Invasix, Israel) deployed on both Caucasian skin types I - III and Asian skin type IV. Histological study demonstrated deep ablation and collagen restructuring in the papillary and reticular dermis. The Fractora device combines the more "cone shaped" ablation seen with $\mathrm{CO}_{2}$ and Erbium lasers with a deep non-ablative heating pattern, seen with other bipolar RF fractional needle resurfacing devices. Ablation, coagulation zones and healing dynamics are analyzed for different energy settings. Two different treatment protocols are suggested: one for light skin and then one for darker skin with a higher risk of post-inflammatory hypperpigmentation. Treatment results show improvement in skin texture, pores, wrinkles and skin dyschromia.
\end{abstract}

Keywords: Skin Resurfacing; Skin Rejuvenation; Fractional Treatment

\section{Introduction}

Since its inception in the 1990's, carbon dioxide $\left(\mathrm{CO}_{2}\right)$ laser resurfacing has enjoyed the distinction of being the gold standard in the treatment of rhytides, texture concerns, acne scars and photo-damage skin. Numerous peer reviewed articles documenting its excellent long-term skin resurfacing results have been reported [1-8]. One of the unique characteristics of the $\mathrm{CO}_{2}$ laser is that it creates almost equal degrees of ablation, coagulation and residual sub-necrotic thermal zones in the skin with volumes of 50 - 100 microns [3]. Although full surface $\mathrm{CO}_{2}$ laser ablation provides some highly regarded results in the treatment of wrinkles, acne scars and photo-damage, it also presents some disadvantages. These problems include a long recovery period, prolonged erythema, uncertain wound healing characteristics derived from the re-epithelialization process, post-inflammatory hyperpigmentation in darker skin types, and long-term hypopigmentation in the majority of patients [9-12].

In response to the disadvantages of ablative $\mathrm{CO}_{2}$ and Erbium YAG lasers, the era of non-ablative near-infrared wavelength lasers and radiofrequency devices arose for sub-necrotic tissue heating inducing collagen remodeling $[13,14]$. All of these non-ablative technologies worked

${ }^{*}$ Corresponding author. through targeting the relatively thick dermal layer of skin, sometimes in combination with external cooling, to preserve the epidermal skin surface from damage and create significant heating in the sub-dermal space. With the correct parameters and wavelengths, the temperature in the dermis can be very close to necrotic levels, inducing collagen remodeling and delivering modest results for skin tightening and wrinkle reduction. With the "non-ablative" approach to skin enhancement, multiple treatments were usually required and results were generally less dramatic than ablative therapies. Clinical data suggests that RF based devices provide stronger tightening effect [15-18], through the ability to penetrate more deeply into the sub-dermal space, while laser penetration depth is limited by the dermis.

Fractional skin resurfacing with non-ablative near infrared laser wavelengths were introduced in 2004, in an effort to deliver high non-ablative columns of energy into the dermis, creating micro-coagulation zones and collagen remodeling, but leaving a significant portion of the adjacent skin untreated to act as a healing reservoir of cells for the non-ablative micro-coagulation zones [19]. The first of the fractional devices were in the near-to midinfrared wavelengths range including $1550 \mathrm{~nm}$ Erbium lasers, $1320 \mathrm{~nm}$ and $1440 \mathrm{~nm}$ wavelengths Nd: YAG la- 
sers [19-21].

In order to produce more significant results than could be achieved with fractional non-ablative coagulative devices, the ablative technologies, like $\mathrm{CO}_{2}$, used a fractionated approach to create ablation columns with depth up to $2 \mathrm{~mm}$. These fractional ablative lasers provided the outcome advantages of ablative technique, but with improved recovery and minor risk of side effects. Fractional $\mathrm{CO}_{2}$ and other fractionally ablative wavelengths have shown excellent improvements in wrinkles, photo-aging and acne scars [22-27]. With the advent of ablative fractional $\mathrm{CO}_{2}$, Thulium and Erbium ablative lasers, dramatic improvement in skin discoloration, texture, wrinkles and acne scars with short downtime and low rate of side effects can be achieved in a single treatment with only several days of recovery time or no downtime and multiple lower fluence treatments.

More recently, alternative ablative technology was introduced that fractionates radiofrequency (RF) energy for skin resurfacing. It creates a low density fractional epidermal and superficial dermal coagulation under the conductive pins and delivers RF travelling through the reticular dermis combining a low density ablative effect in the epidermis with sub-necrotic heating in deeper layers of the skin [28]. This fractional RF approach, with relatively low impact epidermal-junctional effect and deep dermal effect, has been coined fractional "sublative resurfacing". This relatively comfortable treatment demonstrates improvement in lines, acne scars and pigmentation after multiple treatment sessions. The technology compromises with treatment efficiency in comparison to deep ablative fractional lasers, but due to deeper collagen remodeling delivers consistent results after a number of non-aggressive treatment sessions and is particularly good for tissue tightening and some results in acne scar treatment.

This paper reviews the results of using a new fractional RF ablative technology, Fractora (Invasix Ltd.), on Caucasian skin types I - III and Asian skin type IV. Fractora introduces RF energy to the reticular dermis through ablative craters that reproduce the ablative effects of a fractional $\mathrm{CO}_{2}$ laser and an expanded zone of sub-necrotic dermal heating seen with the non-ablative lasers and RF devices. The Fractora combines in one treatment the histological and clinical benefits of epidermal-dermal dominant ablative "cone" type laser resurfacing with the pyramidal shape sublative dermal effect seen with other fractional RF systems. The combined effect of the new technology does not appear to be limited only to skin tightening, wrinkles and acne scar improvement, but initial work appears to provide a synchronous fractional RF improvement in melanin pigmentation, facial telangiectasia, and active acne. The fractional RF device described in this article may also serve to deliver ablative and rejuvenative outcomes in more difficult dyschromia prone skin type IV patients.

\section{Materials and Methods}

The Fractora hand piece used in this study is powered by the BodyTite platform (Invasix, Israel). The Fractora hand pieces come in an array of sharp RF conductive needles. Each needle is $600 \mu$ long and $100 \mu$ wide. There is a 60 pin tip array that provides $10 \%$ surface coverage and a 20 pin tip for the lower lid, upper lid, lip lines and vascular lesions. RF energy per pin and repetition rate is set on the interface prior to treatment. Energy per pin was varied depending on the skin type and thickness. For light skin types I - III and thick skin $50-62 \mathrm{~mJ} /$ pin was applied, while for darker skin type IV patients and thinner skin 10 $40 \mathrm{~mJ} /$ pin was used.

With the Fractora electrode geometry (Figure 1), in addition to the typical "cone shaped" ablative injury seen with $\mathrm{CO}_{2}$, Fractora pins also acts as an array of multiple bipolar electrodes and each sends RF energy through the whole thickness of the papillary and reticular dermis, from the tip of the ablative pin effects to the side flat rail electrodes. This non-ablative, non-specific dermal matrix heating adds a very significant potential of additional skin tightening, that one does not receive with traditional laser fractional ablative treatment. The resulting "double injury" of this device is combination of a "cone shaped" superficial epdiermal-dermal ablation seen with $\mathrm{CO}_{2}$, combined with a dense woven matrix of deep dermal heating in a pyramidal distribution (Figure 1).

20 Caucasian (skin types I and II) and 30 Asian patients (skin types III and IV) with an age range of 21 - 70 years old, received a single full face, ablative fractional radiofrequency treatment using the Fractora hand piece. All patients were observed 3 and 6 months following treatment. For pain control some Caucasian patients treated with high parameters $(40-60 \mathrm{~mJ} /$ pin) underwent

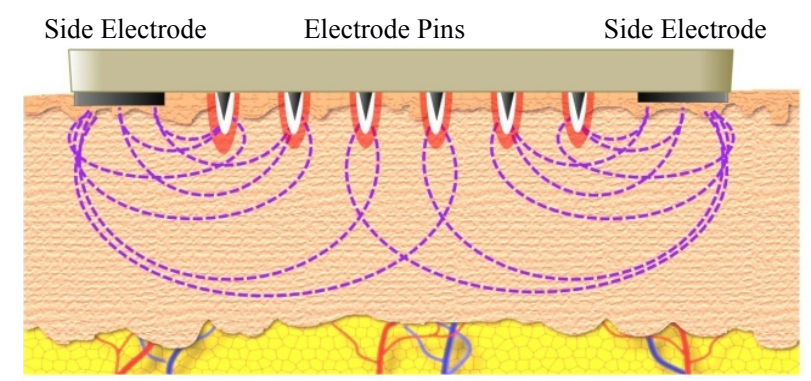

Figure 1. Schematic distribution between pins and side electrodes. The Fractora pins create a " $\mathrm{CO}_{2}$ like injury" with a zone of vaporization (ablation), zone of irreversible coagulation (red) and zone of non-specific thermal stimulation (purple). 
subcutaneous hypodermal tumescent infiltrative anesthesia with a mixture of 1 bottle of $1 \%$ lidocaine mixed in 500 $\mathrm{ml}$ of Ringers lactate and $1 \mathrm{ml}$ of epinephrine 1:1000. Approximately $150 \mathrm{cc}$ of infiltrate was used in the hypodermal space of the brow, cheek and lower face and another $100 \mathrm{cc}$ if the neck was treated. Prior the tumescent hypodermal infiltrative anesthesia, supra-orbital, infraorbital, zygomatical facial, temporal, and mental nerve blocks were performed with $10 \mathrm{cc}$ of $1 \%$ xylocaine. At medium settings $(30-40 \mathrm{~mJ} / \mathrm{pin})$ nerve blocks, topical anesthesia and a Zimmer cold air cooler were used, while at low energy settings $(10-30 \mathrm{~mJ} / \mathrm{pin})$ pretreatment was performed with a topical analgesia and a Zimmer air cooler, or no anesthesia at all.

A single pass, ablative fractional RF treatment was applied using the 60 pin Fractora hand piece. For those regions with deeper rhytides, such as the upper lips, lower lids or acne scars, a second pass was delivered for patients with lighter skin. Smaller areas such as the lower lid, upper lid and the area around the nose, was treated with the 20 pin tip.

RF energy settings used for Asian and Caucasian patients differed significantly. For Caucasian patients, the energy per pin was varied depending on skin type and thickness. For light and thick skin types I - III patients, $50-62 \mathrm{~mJ} /$ pin was applied, while for darker skin type IV and thinner skin patients, $30-40 \mathrm{~mJ} /$ pin was used. In most cases a single treatment was effective enough at high energy settings to achieve the treatment objectives.

For Asian patients the energy per pin was varied in the range of $10-20 \mathrm{~mJ} / \mathrm{pin}$ at the first treatment and was gradually increased up to $30-40 \mathrm{~mJ} /$ pin over the next 3 treatments, performed once a month intervals. It was discovered that after several treatments the skin tone of skin type IV patients became lighter and more uniform, allowing for the application of higher energies at subsequent treatments. For lighter skin tones and younger patients, higher energy was used, while more conservative parameters were selected for darker and older patients.

Antibiotic ointment was applied immediately after the office based treatment. At home, Aquaphor was used to keep the skin moist and until the fractional injury had healed over (usually at $2-5$ days) at which camouflage make-up could be applied. Standard Herpes Simplex prophylaxis consisting of $500 \mathrm{mg}$ of Valtrex orally twice daily for 7 days was used. Standard photographs were taken prior to the treatment and at the 6-month follow up appointments. Patients were advised to take a few days off following the treatment.

Following the treatment and depending upon the parameters, intense edema and erythema were observed and lasted for several hours and up to 1 week, with a minor degree of edema being observed for up to 2 weeks. Small crusted dots, representing the ablated epidermal-dermal tissue at the opening of the ablative crater, appeared the next day following the treatment and were observed up to 1 - 2 weeks after the procedure before flaking off.

Histological samples were taken immediately after treatment and at 1 week, 2 and 6 weeks post treatment to analyze character of fractional lesions and the wound healing process. Samples stained with hematoxylin and eosin show classic signs of fractional carbon dioxide ablative coagulated tissue (a), phagocytosis and healing (b) and collagen remodeling (c). Because different groups of patients are required for different treatment parameters, the histological study consists of 3 energy settings: $10 \mathrm{~mJ} / \mathrm{pin}$, $30 \mathrm{~mJ} /$ pin and $60 \mathrm{~mJ} / \mathrm{pin}$.

Histology images shown in Figure 2 presents treatment results and healing process after the Fractora fractional ablative RF treatment with energy settings of $60 \mathrm{~mJ} / \mathrm{pin}$. Histology of this treatment demonstrated a typical, strong, "cone shaped" epidermal-dermal dominant ablation effect as shown in Figure 2(a). The ablation crater depth is about $500-600 \mu$ in depth, going through the epidermis, the papillary dermis to the mid- and deep reticular dermis. The architecture and shape of the zone of ablation is a typical "cone shaped" lesion (smaller at the base), also seen with $\mathrm{CO}_{2}$ ablative lasers. The zone of coagulation measures 60 - $100 \mu$ surrounding ablation crater (Figure 2(a)).

Figure 2(b) shows phagocytosis in the healing crater (zone of ablation) at 1 week follow-up. Figure 2(c) shows the treated zone structure at 6 weeks post treatment. The phagocytosis is mostly completed and initiation of new collagen formed in the healed crater and around.

At medium energy settings the ablation crater is more shallow, reaching $300-400 \mu$ in depth (Figure 3(a)). When using lower energy the ablation crater ablative depth is approximately $200-250 \mu$ (Figure 4(a)). Similarly, phagocytosis is less active when using lower energy settings (Figure 3(b)) and is completed after one week when using minimal (Figure 4(b)), indicating much faster healing. Collagen remodeling is observed even at minimal energy (Figure 4(c)), but it is more superficial.

Highest energy histologies taken 1 week following treatment is presented in Figure 5. The histology shows the skin with multiple fractional zones and collagen contraction and remodeling in the deep dermal space between the craters, supporting the effect of RF sub-necrotic heating through the entire dermal matrix, from the RF current that flows from the ablative pins to the side rail electrodes. This pyramidal zone of sub-necrotic collagen tightening and remodeling is reflective of the "sublative effects" seen with other fractional RF pin technologies 

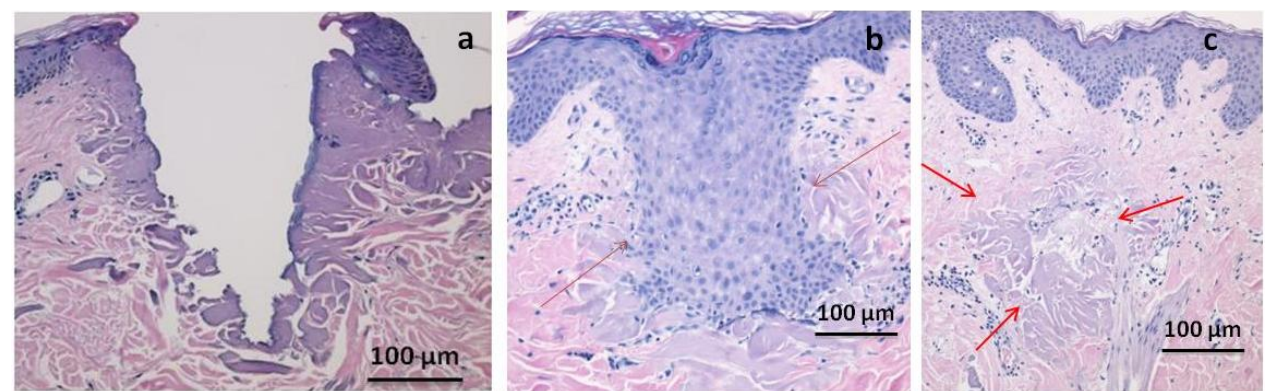

Figure 2. Zone treated with $60 \mathrm{~mJ} /$ pin immediately after the treatment (a), 1 week after the treatment (b) and 2 weeks following the procedure (c).
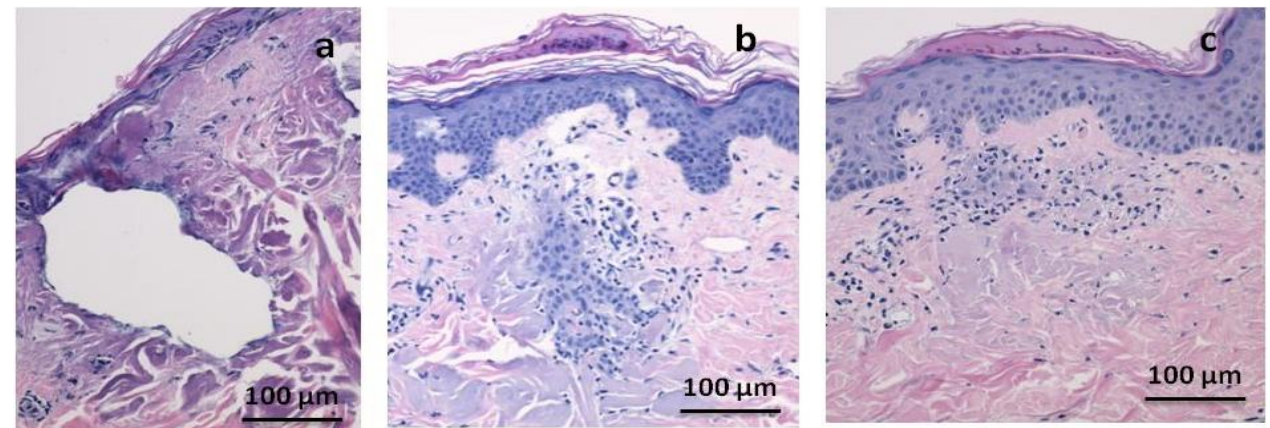

Figure 3. Histological images of a zone treated with $30 \mathrm{~mJ} /$ pin showing results immediately after the treatment (a), 1 week after the treatment (b) and 2 weeks following the procedure (c).
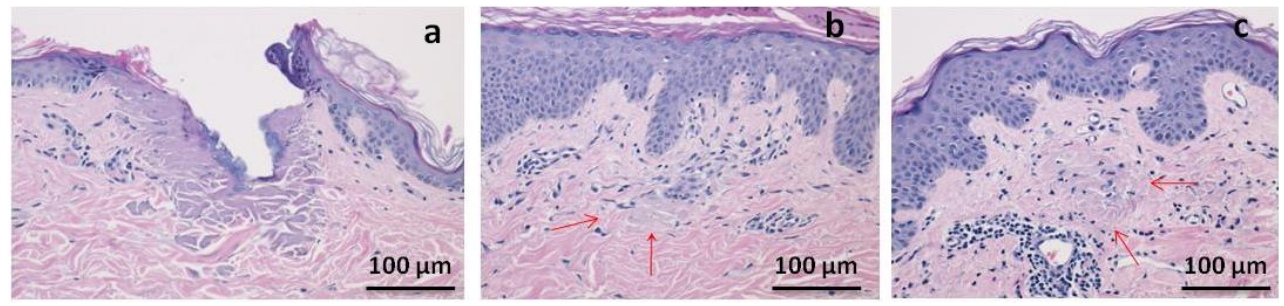

Figure 4. Images capture of a zone treated with $10 \mathrm{~mJ} /$ pin immediately after the treatment (a), 1 week after the treatment (b) and 2 weeks following the procedure (c).

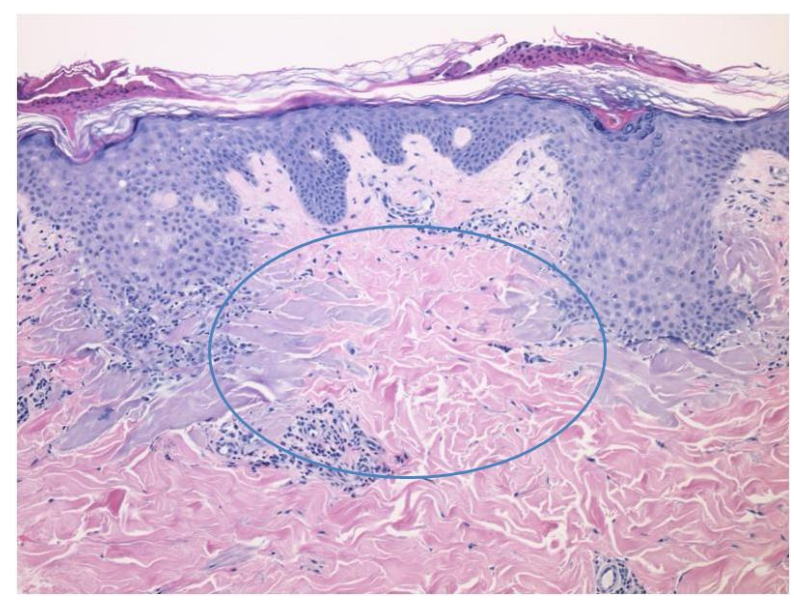

Figure 5. Histology of skin treated with $60 \mathrm{~mJ} / \mathrm{pin}$ at 1 week after the treatment showing collagen restructuring in the space between the two healing craters.
[28]. Fractora is unique in that it combines the strong epidermal "cone shaped" ablation of carbon dioxide with the deep non-ablative tightening, or "sublative" effects seen with fractional RF devices that have a relatively low epidermal impact.

Treatment results and before and after photographs of each patients individual improvement were analyzed and scored using software driven photographic analogue scoring devices for the following clinical concerns:

- Tightening of lax skin;

- Improvement in wrinkles and lines;

- Skin texture improvement;

- Reduction in pore size;

- Improvement in pigmented lesions and skin tone;

- Reduction in facial telangiectasia;

- Acne scar improvement.

From 50 patients' satisfaction survey we had 1 patient 
non-satisfied with treatment results, 10 patients mildly satisfied, 14 patients satisfied, 18 patients happy with results and 7 patients extremely happy.

Figure 6 shows a female patient before and 4 months after a full-face Fractora treatment. The Fractora treatment was delivered at $60 \mathrm{~mJ} / \mathrm{pin}$ and the before and after photographs demonstrate skin tightening, peri-oral wrinkle reduction and an overall improvement of skin quality. Figure 7 shows a Caucasian patient before and 6 months after a full-face Fractora treatment. The energy delivery was $60 \mathrm{~mJ} /$ pin and the treatment demonstrated a significant reduction of pigmented lesions (comparable with an IPL treatment), reduction of peri-oral lines and significant improvement of skin quality. Figure 8 shows a male patient before and immediately after Fractora treatment on vascular lesions. Figure 9 illustrates an Asian 50 years old male with skin type IV before the treatment and 3 months following the last treatment. The patient received 2 treatments with a gradual energy increase from $10 \mathrm{~mJ} /$ pin at the first treatment up to $25 \mathrm{~mJ} / \mathrm{pin}$ at the second treatment. Improvement of peri-orbital lines, skin texture and skin tightening was indicated 3 months following the last treatment. Figure 10 shows a 28 years old skin type IV Asian patient with acne scars before the treatment and 3 months following the last treatment. The patient received 4 treatments where energy was gradually increased from $30 \mathrm{~mJ} /$ pin up to $45 \mathrm{~mJ} / \mathrm{pin}$.

The Visia system (Canfield Inc.) and Korean analog Aramo TS skin diagnostic device (Aram Huvis Co. Ltd.) were used for quantitative analysis of treatment results.

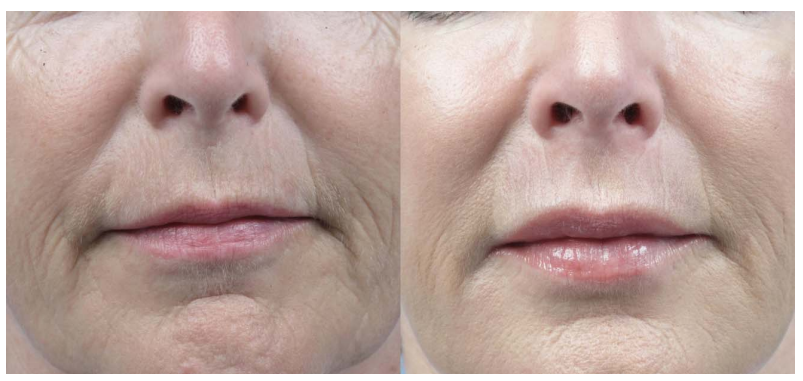

Figure 6. Caucasian female patient before and 4 months after a single full face Fractora treatment.

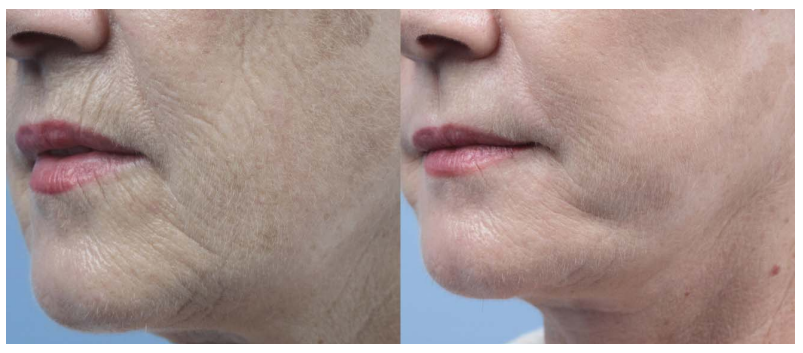

Figure 7. Female patient before and 6 months after a single full face Fractora treatment.

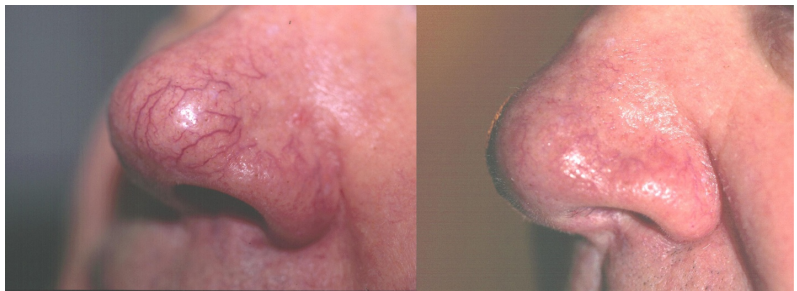

Figure 8. Caucasian patient with vascular lesions (left) and immediate improvements after treatment (right).
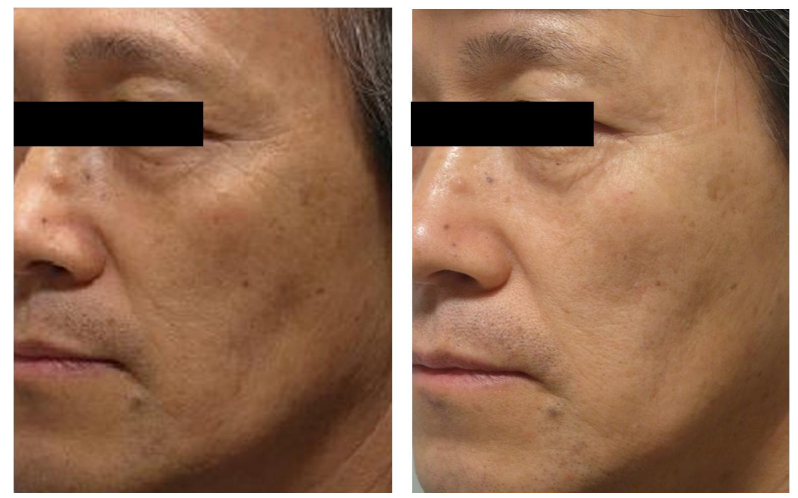

Figure 9. Asian male patient with aged skin before (left) and 3 months after the treatment (right).

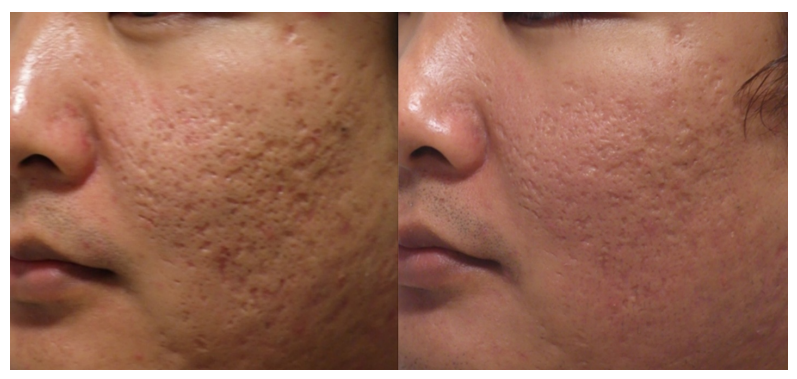

Figure 10. A 28-year-old Asian male before (left) and 3 months after the last treatment (right).

The results are presented in Table $\mathbf{1 .}$

\section{Conclusions}

This study confirms the efficacy and safety of a novel RF fractional ablative device. The histological results confirm the compatibility of Fractora treatment outcomes and many of the clinical skin enhancement that are seen with fractional $\mathrm{CO}_{2}$ resurfacing. The Fractora has the additional benefits of a strong, bipolar RF non-ablative, noncoagulative dermal matrix stimulation not seen with ablative lasers. Fractora also reduces superficial and middermal melanin pigmentation. We also documented a reduction of facial telangiectasia in Caucasian patients, however future studies are recommended on a higher number of patients to fully assess the vascular reduction and parameters required. The review of patients $3-6$ months 
Table 1. Visia and Aramo TS skin diagnostic measured improvements in light and dark skin type patients.

\begin{tabular}{lcc}
\hline Indication & Average Improvement Asian Skin (skin types III and IV) & Average Improvement Caucasian Skin (skin types I and II) \\
\hline Texture & $70 \%$ & $67 \%$ \\
Pores & $40 \%$ & $22 \%$ \\
Wrinkles and lines & $45 \%$ & $63 \%$ \\
Acne scars & $40 \%$ & $40 \%$ \\
Pigmentation & $30 \%$ & $60 \%$ \\
\hline
\end{tabular}

following treatment confirms that the Fractora delivers significant improvements in wrinkles, laxity, brown pigmentation, skin tightening and acne scars.

The safety profile was excellent with no significant adverse events. Patients with skin type IV appear as suitable candidates for treatment using the graduated low fluence parameters set out in this paper without an excessive risk of post inflammatory hyper-pigmentation (PIH) or hypo-pigmentation when receiving gradual energy increase over the series of treatment sessions or accompanied with melanocyte suppression management regimen prior and post treatment.

Fractional ablative RF treatment with a deep needle based delivery system is a novel and unique fractional, ablative technology that provides a total skin rejuvenation solution:

1) Stimulates and produces the wrinkle reduction and skin tightening seen with ablative fractional $\mathrm{CO}_{2}$ resurfacing;

2) Provides additional non-ablative, bipolar dermal matrix thermal stimulation which provides additional skin tightening similar to non-ablative RF devices;

3) Improves pigmented lesions and dyschromia similar to intense pulsed light;

4) Potentially improves superficial vascular lesions (additional study with higher statistics is required to determine consistency).

All patients re-epithelialized within 4 - 7 days. There were no cases or instances of delayed healing, no significant adverse reactions and specifically, no hypo-pigmentation, PIH and no hypertrophic/hypotrophic scars.

The fractional RF multi-needle technology appears to be a suitable for facial treatment of both Caucasian and Asian patients.

\section{REFERENCES}

[1] R. E. Fitzpatrick, W. D. Tope, M. P. Goldman and N. M. Satur, "Pulsed Carbon Dioxide Laser, Trichloroacetic Acid, Baker-Gordon Phenol, and Dermabrasion: A Comparative Clinical and Histologic Study of Cutaneous Resurfacing in a Porcine Model," Archives of Dermatology, Vol. 132, No. 4, 1996, pp. 469-471. doi:10.1001/archderm.1996.03890280135023

[2] D. B. Apfelberg, "The Ultrapulse Carbon Dioxide Laser with Computer Generator Automatic Scanner for Facial Cosmetic Surgery and Resurfacing," Annals of Plastic Surgery, Vol. 36, No. 5, 1996, pp. 522-529. doi:10.1097/00000637-199605000-00015

[3] T. S. Alster, A. N. Kauvar and R. G. Geronemus, "Histology of High-Energy Pulsed $\mathrm{CO}_{2}$ Laser Resurfacing," Seminars in Cutaneous Medicine and Surgery, Vol. 15, No. 3, 1996, pp. 189-193. doi:10.1016/S1085-5629(96)80010-1

[4] D. B. Apfelberg, "Ultrapulse Carbon Dioxide Laser with CPG Scanner for Full-Face Resurfacing for Rhytides, Photoaging, and Acne Scars," Plastic \& Reconstructive Surgery, Vol. 99, No. 7, 1997, pp. 1817-1825. doi:10.1097/00006534-199706000-00003

[5] T. X. Alster and T. B. West, "Resurfacing of Atophic Facial Acnes Scars with a High-Energy, Pulsed Carbon Dioxide Laser," Dermatologic Surgery, Vol. 22, No. 2, 1996, pp. 151-154. doi:10.1016/1076-0512(95)00460-2

[6] D. B. Apfelberg, "A Critical Appraosal of High-Energy Pulsed Carbon Disoxide Laser Resurfacing for Acne Scars," Annals of Plastic Surgery, Vol. 38, No. 2, 1997, pp. 95100. doi:10.1097/00000637-199702000-00001

[7] T. X. Alster and S. Garg, "Treatment of Facial Rhytides with a High-Energy Pulsed Carbon Dioxide Laser," Plastic \& Reconstructive Surgery, Vol. 98, No. 5, 1996, pp. 791-794. doi:10.1097/00006534-199610000-00005

[8] E. V. Ross, J. R. McKinlay and R. R. Anderson, "Why Does Carbon Dioxide Resurfacing Work? A Review," Archives of Dermatology, Vol. 135, No. 4, 1999, pp. 444-454. doi:10.1001/archderm.135.4.444

[9] L. J. Bernstein, A. N. Kauvar, M. C. Grossman and R. F. Geronemus, "The Short- and Long-Term Side Effects of Carbon Dioxide Laser Resurfacing," Dermatologic Surgery, Vol. 23, No. 7, 1997, pp. 519-525.

[10] C. A. Nanni and T. S. Alster, "Complications of Carbon Dioxide Laser Resurfacing: An Evaluation of $500 \mathrm{~Pa}-$ tients," Dermatologic Surgery, Vol. 24, No. 3, 1998, pp. 315-320. doi:10.1016/S1076-0512(97)00510-4

[11] W. Manuskiatti, R. E. Fitzpatrick and M. P. Goldman, "Long-Term Effectiveness and Side Effects of Carbon Dioxide Laser Resurfacing for Photoaged Facial Skin," Journal of the American Academy of Dermatology, Vol. 40, No. 3, 1999, pp. 401-411. doi:10.1016/S0190-9622(99)70489-5 
[12] R. J. Schwartz, A. J. Burns, R. F. Rohrich, R. E. Barton and H. S. Byrd, "Long Term Assessment of $\mathrm{CO}_{2}$ Facial Laser Resurfacing: Aesthetic Results and Complications," Plastic \& Reconstructive Surgery, Vol. 103, No. 2, 1999, pp. 593-601. doi:10.1097/00006534-199902000-00037

[13] N. S. Sadik, "Update on Non-Ablative Light Therapy for Rejuvenation: A Review," Lasers in Surgery and Medicine, Vol. 32, No. 2, 2003, pp. 120-128. doi:10.1002/lsm.10127

[14] M. R. Alexiades-Armenakas, J. S. Dover and K. A. Arndt, "The Spectrum of Laser Skin Resurfacing: Non-Ablative, Fractional and Ablative Laser Resurfacing," Journal of the American Academy of Dermatology, Vol. 58, No. 5, 2008, pp. 719-737. doi:10.1016/j.jaad.2008.01.003

[15] D. J. Hodgkinson, "Clinical Applications of Radiofrequency: Nonsurgical Skin Tightening (Thermage)," Clinics in Plastic Surgery, Vol. 36, No. 2, 2009, pp. 261-268. doi:10.1016/j.cps.2008.11.006

[16] R. A. Weiss, M. A. Weiss, G. Munavalli and K. L. Beasley, "Monopolar Radiofrequency Facial Tightening: A Retrospective Analysis of Efficacy and Safety in over 600 Treatments," Journal of Drugs in Dermatology, Vol. 5, No. 8, 2006, pp. 707-712.

[17] C. S. Yu, C. K. Yeung, S. Y. Shek, R. K. Tse, T. Kono and H. H. Chan, "Combined Infrared Light and Bipolar Radiofrequency for Skin Tightening in Asians," Lasers in Surgery and Medicine, Vol. 39, No. 6, 2007, pp. 471-475. doi:10.1002/1sm.20515

[18] M. Kulick, "Evaluation of a Combined Laser-Radio Frequency Device (Polaris WR) for the Nonablative Treatment of Facial Wrinkles," Journal of Cosmetic and Laser Therapy, Vol. 7, No. 2, 2005, pp. 87-92.

[19] D. Manstein, G. S. Herron, R. K. Sink, et al., "Fractional Photothermolysis: A New Concept for Cutaneous Remodeling Using Microscopic Patterns of Thermal Injury," Lasers in Surgery and Medicine, Vol. 34, No. 5, 2004, pp. 426-438.

[20] R. G. Geronemus, "Fractional Photothermolysis: Current and Future Applications," Lasers in Surgery and Medicine, Vol. 38, No. 3, 2006, pp. 169-176. doi: $10.1002 / 1 \mathrm{sm} .20310$

[21] L. S. Bass, "Rejuvenation of the Aging Face Using Fraxel Laser Treatment," Aesthetic Surgery Journal, Vol. 25, No. 3, 2005, pp. 307-309. doi:10.1016/j.asj.2005.03.003

[22] E. M. Graber, E. L. Tanzi and T. S. Alster, "Side Effects and Complications of Fractional Laser Photothermolysis: Experience with 961 Treatments," Dermatologic Surgery, Vol. 34, No. 3, 2008, pp. 301-305. doi:10.1111/j.1524-4725.2007.34062.x

[23] J. D. Goldberg, A. L. Berlin and R. Phelps, "Histologic and Ultrastructural Analysis of Melasma after Fractional Resurfacing," Lasers in Surgery and Medicine, Vol. 40, No. 2, 2008, pp. 134-138. doi:10.1002/lsm.20591

[24] D. Daniel, L. J. Bernstein, R. G. Geronemus, A. M. Chapas, L. Brightman, S. Sukal and E. Hale, "Successful Treatment of Acneiform Scarring With $\mathrm{CO}_{2}$ Ablative Fractional Resurfacing," Lasers in Surgery and Medicine, Vol. 40, No. 6, 2008, pp. 381-386. doi:10.1002/1sm.20659

[25] N. P. Chan, S. G. Ho, C. K. Yeung, S. Y. Shek and H. H. Chan, "Fractional Ablative Carbon Dioxide Laser Resurfacing for Skin Rejuvenation and Acne Scars in Asians," Lasers in Surgery and Medicine, Vol. 42, No. 9, 2010, pp. 615-623. doi:10.1002/1sm.20974

[26] B. M. Hantash, V. P. Bedi, B. Kapadia, Z. Rahman, K. Jiang, H. Tanner, K. F. Chan and C. B. Zachary, "In Vivo Histological Evaluation of a Novel Ablative Fractional Resurfacing Device," Lasers in Surgery and Medicine, Vol. 39, No. 2, 2007, pp. 96-107. doi:10.1002/1sm.20468

[27] A. M. Chapas, L. Brightman, S. Sukai, et al., "Successful Treatment of Acneiform Scarring with $\mathrm{CO}_{2}$ Fractional Resurfacing," Lasers in Surgery and Medicine, Vol. 40, No. 6, 2008, pp. 381-386. doi:10.1002/1sm.20659

[28] G. Hruza, A. F. Taub, S. L. Collier, S. R. Mulholland, "Skin Rejuvenation and Wrinkle Reduction Using a Fractional Radiofrequency System," Journal of Drugs in Dermatology, Vol. 8, No. 3, 2009, pp. 259-265. 